\title{
Brain Image Compression Techniques
}

\author{
M.Abo-Zahhad ${ }^{\# 1}$, R.R.Gharieb ${ }^{\# 2}$, Sabah M.Ahmed ${ }^{\# 3}$, Mahmoud Khaled ${ }^{* 4}$

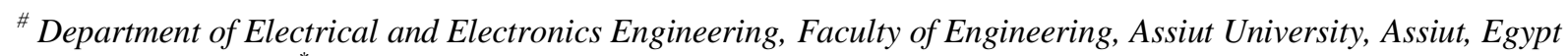 \\ "Madina Higher Institute for Engineering and Technology, Giza, Egypt
}

\begin{abstract}
This paper presents a survey of different methods in the prospect of medical and brain image compression. Compressing an image is basically a process of encoding the image to reduce the size of image as a number of bytes for storage and transmission purposes. This should be done while preserving as much as possible the quality of the image. Hence, image compression has been founded necessary in different medical imaging systems, where there are too much number of bytes of the reconstructed images. There are basically two categories of compression techniques; namely lossless and lossy compression techniques. As the name indicates, in the lossless technique the image is encoded without any loss of information. But in the lossy one, it is allowed that some information is missed. The lossy compression techniques are commonly applied to multimedia data such as audio, video, and still images. Several lossless and lossy compression approaches have been applied to medical images. Different compression approaches belonging to the two categories are discussed and Brain images compression techniques are highlighted. Furthermore, quantitative comparisons between different compression methods are given.as well as advantages and disadvantages of each method.
\end{abstract}

Keywords - Medical image compression, Brain image compression, EEG, CT, Run length encoding, Huffman encoding, LZW, DCT, DWT.

\section{INTRODUCTION}

A number of methods have been presented over the years to perform image compression. They all have one common goal to alter the representation of information contained in an image so that it can be represented sufficiently well with less information [1]. Medical image processing and compression have become an important tool for diagnosis and treatment of many diseases. There are a number of techniques and methods that have been approved and proposed in this regard. However, there is still a need for more work especially with the advancement of medical imaging systems. Bain diseases that can be handled through medical imaging have got advantages from image processing and compression. Nowadays many hospitals around the world have routinely used medical image processing and compression tools.

The basic objective of this study is to review several image compression approaches that have been commonly applied to different medical images with focusing on brain images. These methods are explained and quantitative comparisons with advantages as well as disadvantages of these methods are given.

\section{Main IMAge Compression CATEgORIES}

The need for data storage capacity and transmission bandwidth continues to exceed the capability of available technologies. The process of obtaining a compact representation of an image while maintaining all the necessary information important for medical diagnosis is referred to as image compression. Image compression is basically a process of reducing the size in bytes of images deprived of demeaning the superiority and quality of the image to an objectionable level. The decrease in image size permits further images to be deposited in a specified quantity of disk or memory space, in order to present a part of human body in digital form. The image compression methods are generally categorized into two central types: Lossless and Lossy methods. The major objective of each type is to rebuild the original image from the compressed one without affecting any of its numerical or physical values [2].

\section{A. Lossless Compression Techniques}

In the lossless compression techniques, the original image can be perfectly recovered from the compressed (encoded) image. These are also called noiseless because since there compression process is perfectly reversible (i.e., there is no error noise). It is also known as entropy coding since it uses statistical decomposition techniques to eliminate or minimize redundancy in the original image. Lossless compression is therefore a demand however, due to the cost, it is used only for a few applications with stringent requirements such as medical imaging. Various lossless compression algorithms have been proposed and used. This techniques include Run Length Encoding, the Huffman Coding, Arithmetic Encoding and Dictionary Based Encoding [3]. Following techniques are included in lossless compression:

1) Run Length Encoding: Run length Encoding (RLE) is basically based on the idea of encoding a consecutive occurrence of the same symbol. This is achieved by replacing a series of repeated symbols with a count and the symbol. That is, RLE finds the number of repeated symbols in the input image and replaces them with two-bytes code. The first byte for the number and the second one is for the symbol. For a simple illustrative example, the string 'AAAAAABBBBCCCCC' is encoded as 'A6B4C5'; that saves nine bytes (i.e. compression ratio $=15 / 6=5 / 2$ ). However in some cases there is no much consecutive repeation which reduces the compression ratio. An illustrative example, the original data " $12000131415000000900 "$, the RLE encodes it to "120313141506902" (i.e. compression ratio $=20 / 15=4 / 3$ ). Moreover if the data is random the RLE may fail to achieve any compression ratio [4]-[5].

2) Huffman encoding: It is the most popular technique for removing coding redundancy. When the symbols of an information source are coded individually, Huffman coding 
yields the smallest possible number of code symbols per source symbols. This method is started with a list of the probabilities of the image data elements. Then, take the two least probable elements and make them two nodes with branches (labeled " 0 " and " 1 ") to a common node which represents a new element. The new element has a probability, which is the sum of the two probabilities of the merged elements. The procedure is repeated until the list contains only one element [6]. Thus statistical occurrence frequencies (probabilities) to carry out the process are used. Each pixel of the image is treated as a symbol. Then histogram then computed to count the frequency occurrence of each symbol.

3) LZW coding: LZW (Lempel- Ziv - Welch) is a dictionary based coding which can be static or dynamic. In static coding, dictionary is fixed during the encoding and decoding processes. In dynamic coding, the dictionary is updated on fly. LZW is widely used in computer industry and is implemented as compress command on UNIX [4].

4) Area coding: Area coding is an enhanced form of RLE, reflecting the two dimensional character of images. This is a significant advance over the other lossless methods. For coding an image, it handles the image as an array of sequences building up a two dimensional object. The algorithms for area coding try to find rectangular regions with the same characteristics. These regions are coded in a descriptive form as an element with two points and a certain structure. This type of coding can be highly effective but it bears the problem of a nonlinear method, which cannot be implemented in hardware. Therefore, the performance in terms of compression time is not competitive, although the compression ratio is in comparison with RLE that deals with two dimensional characters of images [6].

\section{B. Lossy Compression Techniques}

Lossy Compression techniques deliver considerably greater compression percentages than lossless ones. The original and decompressed images are not perfectly matched. However, practically near to each other, in the sense that the difference is random noise. In the following subsections, several Lossy compression techniques are reviewed:

1) Transformation coding: Another approach to image compression is the use of transformations that operate on an image to produce a set of coefficients. A subset of these coefficients is chosen and quantized for transmission across a channel or for storage. The goal of this technique is to choose a transformation for which such a subset of coefficients is adequate to reconstruct an image with a minimum of discernible distortion. The Fourier Transform (FT) is not a practical approach to represent boundaries and edges of objects of an image. However, Wavelet Transform (WT) provides multi resolution analysis in which edges and boundaries can be represented. Since WT and cosine transform have become the most popular technique for image compression, we devote this subsection to review them.

- Discrete Cosine Transform (DCT): The Discrete Cosine Transform (DCT) was first proposed by Ahmed et al.
(1974). It has been more and more important in recent years [7]. The DCT is similar to the discrete Fourier transform that transforms a signal or image from the spatial domain to the frequency domain as shown in Fig. 1.

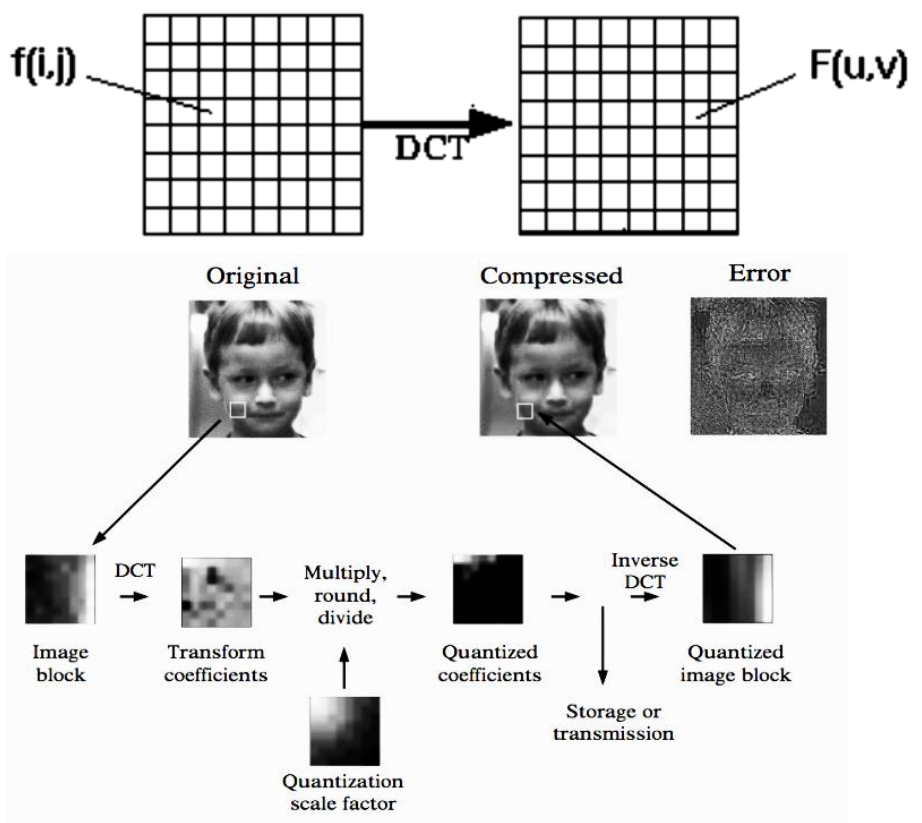

Fig. 1 Image transformation from the spatial domain to the frequency domain [8].

DCT represents a finite series of data points as a sum of harmonics cosine functions. DCTs representation have been used for numerous data processing applications, such as lossy coding of audio signal and images. It has been found that small number of DCT coefficients are capable of representing large sequence of raw data. This transform has been widely used in signal processing of image data, especially in coding for compression, for its near-optimal performance. The discrete cosine transform helps to separate the image into spectral sub-bands of differing importance with respect to the image's visual quality [7]. The use of cosine is much more efficient than sine functions in image compression since function capable of represent edges and boundary. As described below, fewer coefficients are needed to approximate a typical signal.

The Two-dimensional DCT is useful in the analysis of twodimensional (2D) signals such as images. Since the 2D DCT can be computed by applying 1D transforms separately to the rows and columns, we say that the $2 \mathrm{D}$ DCT is separable in the two dimensions. The 2D DCT is computed in a simple way: The 1D DCT is applied to each row of an image, s, and then to each column of the result. Thus, the transform of the image $s$ is given by

$S(u, v)=$

$\frac{2}{\sqrt{n m}} C(u) C(v) \sum_{y=0}^{m-1} \sum_{x=0}^{n-1} s(x, y) \cos \frac{(2 x+1) u \pi}{2 n} \cos \frac{(2 y+1) v \pi}{2 m}$,

Where $u=0,1,2, \ldots, n-1$; and $v=0,1,2, \ldots, m-1$. ( $n x$ $m$ ) is the size of the block that the DCT is applied on. 
Equation (1) calculates one entry $(u, v)$ of the transformed image from the pixel values of the original image matrix [7].

DCT is widely used especially for image compression for encoding and decoding, at encoding process image divided into $\mathrm{N}$ x $\mathrm{N}$ blocks after that DCT performed to each block. Quantization applied to DCT coefficient to compress the blocks so selecting the quantization affect compression value. Compressed blocks are saved in storage with significantly space reduction. In decoding process compressed blocks are loaded which de-quantized with reverse the quantization process. Inverse DCT was applied on each block and merging blocks into an image which similar to original one [68]. For the standard JPEG compression uses a block of 8x8.

- Discrete Wavelet Transform: Two-dimensional Discrete Wavelet Transform (2-D DWT) is devoted for digital images; 2-D data that require a two-dimensional wavelet transform. The 2-D DWT analyzes an image across rows and columns in such a way as to separate horizontal, vertical and diagonal details as shown in Fig. 2. The $\downarrow 2$ donates down sampled by 2 .

Three-dimensional Discrete Wavelet Transform (3-D DWT) 3-D wavelets can be constructed as separable products of 1-D wavelets by successively applying a 1-D analyzing wavelet in three spatial directions (x, y, z). Fig. 2 shows a one-level separable 3-D discrete wavelet decomposition [9] of an image volume. The volume $\mathrm{F}(\mathrm{x}, \mathrm{y}, \mathrm{z})$ is firstly filtered along the $\mathrm{x}$ dimension, resulting in a low-pass image $\mathrm{L}(\mathrm{x}, \mathrm{y}, \mathrm{z})$ and a high pass image $\mathrm{H}(\mathrm{x}, \mathrm{y}, \mathrm{z})$. Both $\mathrm{L}$ and $\mathrm{H}$ are then filtered along the $y$-dimension, resulting in four decomposed sub-volumes: LL, $\mathrm{LH}, \mathrm{HL}$ and $\mathrm{HH}$. Then each of these four sub-volumes is filtered along the z-dimension, resulting in eight sub-volumes: LLL, LLH, LHL, LHH, HLL, HLH, HHL and HHH as shown in Fig. 3.

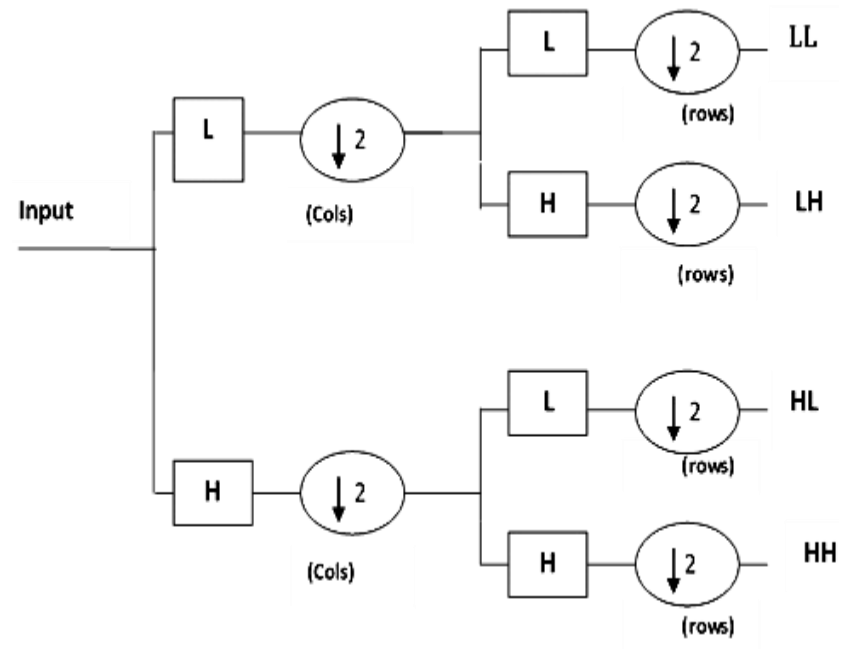

Fig. 2 A one-level two-dimensional DWT decomposition
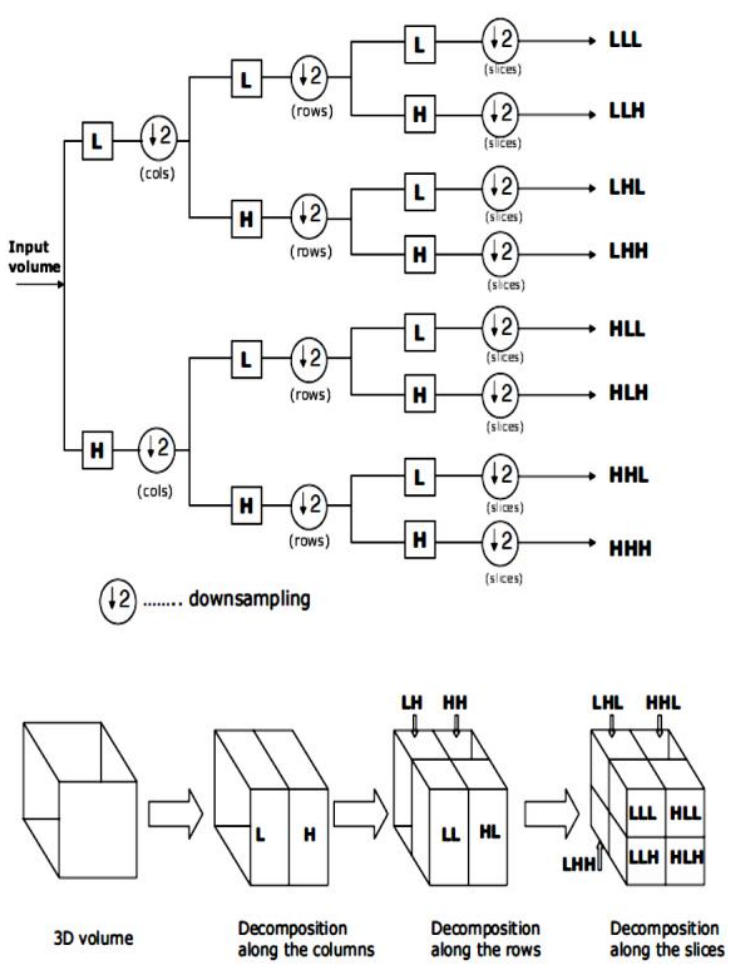

Fig. 3 Three-dimensional DWT decomposition

Dual Tree Complex Wavelet Transform (DT CWT) has significant advantages over real wavelet transform for certain signal processing problems. The 1-D dual-tree wavelet transform is implemented using a pair of filter banks operating on the same data simultaneously as shown in Fig. 4. The upper iterated filter bank represents the real part of a complex wavelet transform. The lower one represents the imaginary part [9]. DWT has been used to comprese an image through encoding process. Image passed through low and high pass filters which applied to each row then the output combined and down sampled by 2. After that filters applied on each column which combined and down sampled by 2 . Which provide a compressed image. For decoding process inverse DWT applied [68].

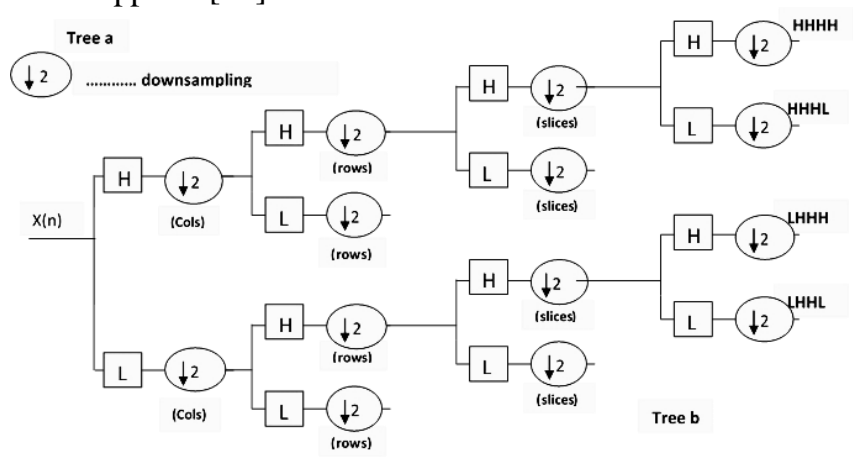

Fig. 4 The 1-D dual-tree complex wavelet transforms

2) Vector quantization $(V Q)$ : In VQ coding phase, the image is subdivided into blocks, typically of a fixed size of $\mathrm{n} \times \mathrm{n}$ pixels. For each block, the nearest codebook entry under the distance metric is found and the ordinal number of the 
entry is transmitted. On reconstruction, the same codebook is used and a simple look-up operation is performed to produce the reconstructed image [10]. The advantage of vector quantization over scalar quantization, in which each pixel is quantized independent of its neighbourhoods, is that the high degree of correlation between neighbouring pixels can be exploited. Even for a memory less system, the coding of vectors instead of scalars can theoretically improve performance. Address Predictive Vector Quantization has been also used in literature for image compression. This technique uses an ordered codebook in which adjacent entries are in some sense correlated. The correlation between adjacent codewords and the correlation between neighbouring blocks in an image can be exploited to construct a DPCM encoder in which the input signal is the code-word address [11].

A Finite State Vector Quantization (FSVQ) is another technique developed for signal compression. In FSVQ the codeword index $i_{n}$ is chosen from a state code-book of the current state $S_{n}$. The current state is a function of the previous state $\mathrm{S}_{\mathrm{n}-1}$ and the previous index $\mathrm{i}_{\mathrm{n}-1}$. If the state transition function is a good predictor of the next input, then each state code-book can be much smaller than that required for a memoryless quantizer [12].

Learning Vector Quantization is a supervised learning algorithm which can be used to modify the codebook if a set of labelled training data is available [13]. For an input vector $\mathrm{x}$, let the nearest code-word index be $\mathrm{i}$ and let $\mathrm{j}$ be the class label for the input vector. The learning-rate parameter is initialized to, say, 0.1 and then decreases monotonically with each iteration. After a suitable number of iterations, the codebook typically converges and the training is terminated.

The main drawback of the conventional VQ coding is the computational load needed during the encoding stage as an exhaustive search is required through the entire codebook for each input vector. An alternative approach is to cascade a number of encoders in a hierarchical manner that trades off accuracy and speed of encoding [14]-[15]. Fig. 5 illustrates this approach which is called Hierarchical Vector Quantization (HVQ). For a bit rate of 2 bits/sample, or equivalently 8 bits per input vector, each stage requires only 24 code-words for a total search space of 32 code-words. Lee and Peterson have developed an approach which dynamically changes the structure and neighbourhood relationships between the weights by allowing creation of new weights, merging and deletion of existing weights, and merging of axes in the topological map [16].

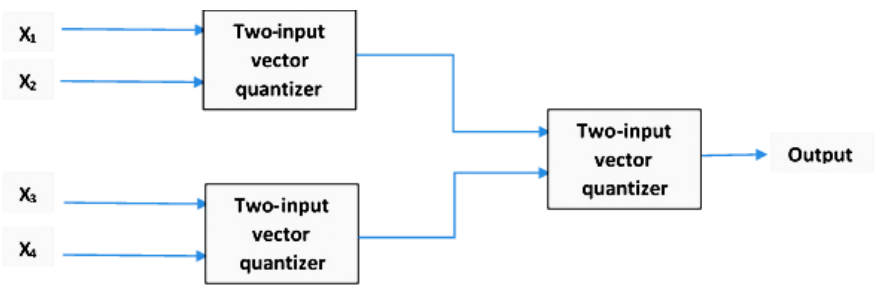

Fig. 5 Two-stage hierarchical vector quantizer using two-input vector quantizer.
3) Fractal coding: Fractal image compression is a process to find a small set of mathematical equations that can describe the image. By sending the parameters of these equations to the decoder, we can reconstruct the original image. In general, the theory of fractal compression is based on the contraction mapping theorem in the mathematics of metric spaces [6].

4) Block truncation coding: In block truncation coding, the image is divided into non overlapping blocks of pixels. For each block, threshold and reconstruction values are determined. The threshold is usually the mean of pixel values in the block. Then a bitmap of the block is derived by replacing all pixels whose values are greater than or equal and less than to the threshold by a 1 and 0 , respectively. Then for each segment there are groups of $1 \mathrm{~s}$ and $0 \mathrm{~s}$ in the bitmap, the reconstruction value is determined as the average of the values of the corresponding pixels in the original block [17].

5) Sub band coding: In this scheme, the image is analyzed to produce the components containing frequencies in well-defined bands, the sub bands. Subsequently, quantization and coding is applied to each of the bands. The advantage of this scheme is that the quantization and coding well-suited for each of the sub bands can be designed separately [17].

\section{MedicAl IMAGE COMPRESSION}

Most hospitals store medical image data in digital form using picture archiving and communication systems due to extensive digitization of data and increasing telemedicine use. If we look inside the medical image processing, we can see that there are many medical issues being treated through this processing. These issues comprise subjects related to heart, brain, lungs, kidney, cancer diagnosis, stomach etc. An effort has been done to provide effective storage of medical images with patient medical record for future use and also for effective transfer between hospitals and health care centers. In the following, the most important medical image compression techniques that have been proposed are reviewed:

-In [18], a method has been developed for a 3-D image compression. It uses a separable non-uniform 3-D wavelet transform. The non-uniform 3-D transform applies one wavelet filter bank in the $\mathrm{x}$ and $\mathrm{y}$ directions, and a second wavelet filter bank in the $\mathrm{z}$-direction. The second wavelet filter bank is selected for image sets at various slice distances according to the best compression performance. In [19] Rodet et al., make use of steps named decomposition, quantification and un-compression based on Fourier decomposition. Hashimoto et al., [20] proposed a system of lossless compression by using the Region of Interest (ROI) method by means of optimal degree, motion remunerated and lossless compression in additional areas. The process works by first applying the segmentation process to the input image. After that motion compensated coding is applied. The next step involves the utilization of entropy minimizing coding of motion vector. The method is tested on CT images. The experimental results showed that $2.5 \%$ rate can be achieved by this compression method. In [21] Kanoun et al, makes use of 


\section{International Journal of Engineering Trends and Technology (IJETT) - Volume 19 Number 2 - Jan 2015}

DCT which is the most common method among the compression methods. The results indicate that it can be applied to different medical modalities together with the feature of image quality preservation factor. A lossless compression method for medical image sequences using JPEG-LS and inter-frame coding is presented in [22]. Another lossless medical images compression method was introduced by Tzong-Jer and Keh-Shih [23]. This method is based on eliminating the noise factor without damaging the image quality. The results of this method show that the use of Haar wavelet transform in the slice direction gives the optimum results in the majority of cases except for the CT image set with 1-mm slice distances.

-In 2012, Dubey and Singh, have proposed 3D medical image compression using Huffman encoding technique, by converting colour image into grey level image. Then the symbols that represent pixel values which is non-repeated are defined. Then the probability of each symbol is calculated and arranged in deciding order. Then these probabilities is divided into groups of two which each group merged into one. This procedure is continued until reaching only two probabilities. The codes are then assigned according to the rule "the highest probable symbol will have a shorter length code". Thus, Huffman encoding is performed [24].

-In [25] image compression technique is performed using bi-orthogonal filters for implementing the wavelet decomposition. The decomposed images are then passed through an $\mathrm{m}$ channel analysis-synthesis filter and the compression is done using FIR-IIR filters [9]-[10].This analysis leads to the horizontal, vertical, approximation and diagonal details. The analysis-synthesis filters are based on the Quadrature Mirror Filters (QMF).

-In [26], Kumar and Goel have presented a technique that accepts input image and produces segmented image as output. It consists of various modules namely preprocessing, and fuzzy segmentation. This method starts with the key frame of

TABLE

\begin{tabular}{|c|c|c|c|}
\hline Reference & Advantages & Limitations & Results \\
\hline [18] & $\begin{array}{l}\text { Image quality is preserved by } \\
\text { removing the noise factor }\end{array}$ & A bit slow system & $\begin{array}{l}\text { Compression rate was enhanced by } 70 \% \text { for } \\
\text { MRI and by } 35 \% \mathrm{CT}\end{array}$ \\
\hline [19] & $\begin{array}{l}\text { Handle the compression ratio and } \\
\text { limitations of signal quality }\end{array}$ & Excellence of signal & $\begin{array}{l}\text { A great compression rate with minimization of } \\
\text { the processing time }\end{array}$ \\
\hline [20] & $\begin{array}{l}\text { Can handle both 2D and 3D medical } \\
\text { image }\end{array}$ & Involves too many processes & Compression ratio of $2.5 \%$ is achieved \\
\hline [21] & Noise removal & Quality of image is affected & $\begin{array}{l}\text { Acceptable and promising compression rates are } \\
\text { achieved }\end{array}$ \\
\hline [22] & Image quality is preserved & $\begin{array}{l}\text { Coding is activated only when } \\
\text { inter frame correlation is high } \\
\text { enough }\end{array}$ & Compression gains: $13.3 \%$ and $26.3 \%$ \\
\hline [23] & Image quality is preserved & Expensive system & $\begin{array}{l}\text { improvement of more than } 40 \% \text { incompression } \\
\text { ratio without deterioration in image quality }\end{array}$ \\
\hline [24] & $\begin{array}{l}\text { has better performance than JPEG } \\
\text { with low and high bit rates. }\end{array}$ & & \\
\hline [25] & success rate of 80 percent & $\begin{array}{l}\text { Small average distortion can } \\
\text { result in a damaging visual } \\
\text { artifact }\end{array}$ & $\begin{array}{l}\text { compression ratios are in the range of } 40 \text { 's and } \\
\text { the PSNR values in the range of 50's }\end{array}$ \\
\hline [26] & $\begin{array}{l}\text { improve the performance of }(\mathrm{CT}) \text { and } \\
\text { (PSNR) }\end{array}$ & Quality of image is affected & \\
\hline
\end{tabular}


-Midtvik and Hovig,1999, have developed MRI compression method using reversible procedure. The method makes use of stagnant based procedures intended for the background and foreground distinctly [28].

-Cavaro-Menard et al., 1999, have developed lossy compression method for MRI. The method makes use of two other methods called JPEG and wavelet transform to compress the MR images [29].

-Wavelet transform has been adopted for MRI compression by many authors; namely Dhouib et al., 1994 [30], Badawy et al., 2002 [31], Karras, 2009 [32], and Hu et al., 2010 [33].

-In 2007 Gornale et al., have developed MRI compression method using a heterogeneous wavelet filter banks. Their method consisting from the following three steps [34].

* Use of decomposition filter that decomposes the image coefficients in each detailed sub-band using wavelet coefficients at selected levels to describe image features.

* The number of coefficients is placed as constant in the preprocessing process.

* The reconstruction of the image is carried out by means of reconstruction filter coefficients.

Numerical results show that the compression ratio is basically depends on the form of image and category of transforms since no filter exists with the intention of performing the most excellent compression for the entire image. Therefore, there is at all times essential to choose the suitable threshold to obtain superior compression and the smallest amount of loss of image contents [34].

-In 2008, Liu and Zhang developed a method based on widening the one dimensional Auto Regression (AR) representation to two dimensional AR model. The method has offered a new sensitivity map assessment system to enlarge the evaluation correctness [35].

-In 2010, Corvetto et al., proposed MRI compression using concept of foreground [36].

-In 2012, G. Soundarya and S. Bhavani, used hybrid coding technique for MRI brain (tumor) images compression. It provides increased compression ratio than existing compression schemes. Their method is based on segmenting the image into ROI and Non-ROI regions. To separate ROI and non-ROI regions, it is required that the image to be segmented. In this scheme, the MRI human brain image is segmented by using Region Growing algorithm and then coded using the hybrid schemes. Numerical experiments show that the hybrid coding techniques resulted in higher compression ratio than existing JPEG 2000 scheme. Hence it proves that the hybrid coding schemes outperform the other existing techniques in compressing the MRI brain images. Fig. 6 depicts the proposed hybrid scheme [37].

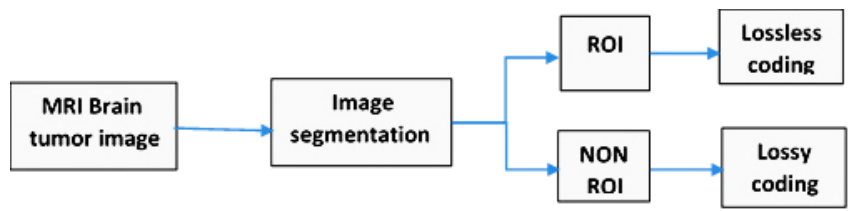

Fig. 6 Hybrid scheme MRI image compression.

-In [38], compression of 3D medical image using EDGE preservation technique was proposed by Alagendran and Manimurugan. In this paper the 3-D medical image is given as input image and it is further undergoing the process of splitting into groups of slices (GOS). Then the 3-D wavelet transform is applied, followed by 3-D encoding. To encode the image and to get them final compressed image, both the wavelet transforms such as symmetric and decoupled wavelet transform are used. 3D DWT has been chosen to preserve the edges. In this algorithm, one level DWT is applied to decompose a 3D low resolution image into eight different subband images. The high frequency coefficients undergo the process of quantification in order to compress the image whereas the low frequency coefficients undergo the Huffman coding process for compressing the image. Thus, the edges of the images are corrected and clear visual effects are obtained.

-Seddeq E. Ghrare, and Salahaddin M. Shreef, 2012, were proposed quality evaluation of compressed MRI medical images for telemedicine applications. In this study; three different magnetic resonance medical images which are brain, spine, and knee have been compressed and reconstructed using wavelet transform. The quality of the resulting compressed images is evaluated using the two most commonly used criteria: objective and subjective measures. The results show that the compression ratio of 40:1 was acceptable for brain image, whereas for spine and knee images 50:1 was acceptable for clinical applications [39].

TABLE II

COMPARISON BETWEEN MRI COMPRESSION TECHNIQUES

\begin{tabular}{|l|l|l|l|}
\hline Reference & Advantages & Limitations & Results \\
\hline$[30]$ & Handles 3D data & $\begin{array}{l}\text { Image storage requirements } \\
\text { are high }\end{array}$ & $\begin{array}{l}\text { The results showed that the method is effective for } \\
\text { compression but requires more memory for } \\
\text { storage }\end{array}$ \\
\hline$[27]$ & $\begin{array}{l}\text { The particulars of the real image are } \\
\text { protected in there built image and are } \\
\text { conserved }\end{array}$ & $\begin{array}{l}\text { Problems of empty cells in } \\
\text { the image do exist }\end{array}$ & $\begin{array}{l}\text { High compression rate is achieved with minimum } \\
\text { utilization of SNR values }\end{array}$ \\
\hline$[28]$ & Fast system & Computationally complex & $\begin{array}{l}\text { The outcomes demonstrated that this compression } \\
\text { method can provide bit rates analogous to the } \\
\text { finest current alterable approaches }\end{array}$ \\
\hline$[29]$ & Also effective for segmentation & $\begin{array}{l}\text { Limiting the compression } \\
\text { ratio }\end{array}$ & The method provides a compression ratio of $67: 1$ \\
\hline
\end{tabular}


International Journal of Engineering Trends and Technology (IJETT) - Volume 19 Number 2 - Jan 2015

\begin{tabular}{|c|c|c|c|}
\hline [31] & Preserves image quality & Large and complex system & Obtained acceptable compression \\
\hline [34] & (Badawyet al., 2002) ratio & & \\
\hline [35] & $\begin{array}{l}\text { Can handle variety of images with } \\
\text { different ranges of frequency and } \\
\text { intensity, Preserves frequency content }\end{array}$ & $\begin{array}{l}\text { Images with higher } \\
\text { frequencies does not } \\
\text { encompass promising } \\
\text { results while slow } \\
\text { frequency contents based } \\
\text { images are only effective } \\
\text { for this method }\end{array}$ & $\begin{array}{l}\text { The compression ratio is based on the kind of } \\
\text { image and category of transforms since no filter } \\
\text { exists that achieves the greatest compression ratio } \\
\text { on behalf of every image, The results showed that } \\
\text { the outcome of the compression procedure usually } \\
\text { rests on the image features }\end{array}$ \\
\hline [32] & Preserves image quality and details & - & $\begin{array}{l}\text { Results showed that proposed method } \\
\text { significantly improves the quality of reconstructed } \\
\text { MR images }\end{array}$ \\
\hline [33] & $\begin{array}{l}\text { Does not require user defined } \\
\text { parameters }\end{array}$ & $\begin{array}{l}\text { The chief area handled in } \\
\text { this case is the removal of } \\
\text { obstructive possessions in } \\
\text { the divider borders }\end{array}$ & $\begin{array}{l}\text { The method obtained auspicious outcomes } \\
\text { regarding recreation process of image excellence } \\
\text { in addition to protection of important image } \\
\text { particulars where as conversely attaining great } \\
\text { compression rates }\end{array}$ \\
\hline [36] & Reliable and effective & Complex system & $\begin{array}{l}\text { Acceptable compression rates by great contrast } \\
\text { with outstanding spatial resolution are attained by } \\
\text { the process }\end{array}$ \\
\hline [37] & Also handles 3D images & - & - \\
\hline [38] & $\begin{array}{l}\text { increased compression ratio than } \\
\text { existing compression }\end{array}$ & $\begin{array}{l}\text { require the image to be } \\
\text { segmented }\end{array}$ & $\begin{array}{l}\text { hybrid coding techniques resulted in higher } \\
\text { compression ratio than existing JPEG } 2000\end{array}$ \\
\hline [39] & $\begin{array}{l}\text { better results than compressed 3D } \\
\text { medical image without edge } \\
\text { preservation }\end{array}$ & Complex system & $\begin{array}{l}\text { have sharper edge features, more details, and } \\
\text { visually it is closer to the original image when } \\
\text { compared to the base paper PSNR results }\end{array}$ \\
\hline
\end{tabular}

\section{B. Computed Tomography Images Compression}

Computed Tomography (CT) is essentially made of investigating dimensions of an image of brain centered on the degree of alteration presence of X-rays. CT process is comprised of examination of the subject being controlled. In the following the most important CT compression techniques, summarized in Table 3, are reviewed.

-Rhodes et al., 1985, have developed method basically preserves the contents of the image. For this purpose they first computed the entropy measures of several hundred CT images by the help of differential streams. Next a method called flexible run-length procedure of compression is applied on the images. Result of this method showed that image entropy procedures were revealed to be an extra complicated compression method as compared to standard entropy [40].

- Lee et al., 1991, have introduced Estimated Inter-frame (DEI) method proposed for the purpose of compression. The results of this method indicated that the process is applicable for both 2D and 3D images [41].

-Hashimoto et al., 2004, have present a study basically describes two techniques. The first technique is the Level of Interest (LOI) technique that is based on giving primacy to the pixels consuming a specific pixel value. The second technique is the Region of Interest (ROI) technique that is based on giving priority to the pixels of a specific area or region within the image while encoding or decoding the image. The main results showed that high-quality compression through lossless process is attained by developed integer technique being proposed [21].

-Another method has been carried out in Li et al., 2006, which preserves the image quality and details [35].
-In $2009 \mathrm{Ju}$ and Seghouane, have introduced method using the concept of spectral frequency [42]. Simulation results showed increase of both reliability and coding efficiency [43].

-In 2010 Sepehrband et al., have introduced a method for lossless compression of CT images. They use transformation method called Differential Pulse Code Modulation (DPCM) where the image is transformed;Huffman encoded, and bit stream compressed. Then it is Huffman decoded and inverse transformed to reconstruct the image. The main results of this method are it is a very simple and accurate method for the compression of CT images [44].

-In 2013, fast and efficient medical image compression technique using contourlet transform was proposed by Kumar, and Goel. In this paper, the authors modified the Embedded Zero tree Wavelet (EZW) algorithm with improved version of contourlet transform for the significant region and DWT for the insignificant region together with fuzzy $\mathrm{C}$-means based segmentation technique. This modified algorithm has two specifications: it distributes entropy differently than the original Shapiro EZW algorithm and it optimizes the coding compared to the existing algorithms. So it accepts input image and produces segmented image as output. It consists of various modules namely preprocessing, and fuzzy segmentation. The proposed system starts with the key frame of the medical image, preprocessing of the image is done for removing the noise for a better segmentation. After preprocessing, segmentation and tracking are performed. A model fitting technique has been applied after tracking the borders. The tracked borders are decomposed into meaningful regional parameters [45]. 
TABLE III

COMPARISON BETWEEN CT IMAGE COMPRESSION TECHNIQUES

\begin{tabular}{|c|c|c|c|}
\hline Reference & Advantages & Limitations & Results \\
\hline [40] & Images are heavily compressed & $\begin{array}{l}\text { Worst case of system exists } \\
\text { that doesnot compress the } \\
\text { image and computationally } \\
\text { expensive }\end{array}$ & The images were compressed $5 \%$ of their original size \\
\hline [41] & $\begin{array}{l}\text { Applicable for both 2D and 3D } \\
\text { data }\end{array}$ & Computationally complex & Effective and efficient method for compression \\
\hline [21] & Adequate image quality is obtained & Computationally expensive & $\begin{array}{l}\text { The whole image has improved eminence and power } \\
\text { of enlightening the excellence ofpixels }\end{array}$ \\
\hline [35] & $\begin{array}{l}\text { Preserves the image quality and } \\
\text { detail }\end{array}$ & $\begin{array}{l}\text { Applicable to a specific } \\
\text { type of images }\end{array}$ & $\begin{array}{l}\text { Meaningful portion of image was compressedand the } \\
\text { process of examiningimage delivers } \\
\text { acceptableoutcomes }\end{array}$ \\
\hline [42] & Image quality is not affected & $\begin{array}{l}\text { Comparably complex } \\
\text { system }\end{array}$ & The method provides $96 \%$ sensitivity for polyps \\
\hline [43] & $\begin{array}{l}\text { Increase of both reliability and } \\
\text { coding efficiency }\end{array}$ & Comparably a slow system & $\begin{array}{l}\text { The algorithm potentials were assessed in terms of its } \\
\text { performance and PET-CT image qualityand the } \\
\text { results are effective ineach regard }\end{array}$ \\
\hline [44] & $\begin{array}{l}\text { Has smallest entropy and very easy } \\
\text { to implement on hardware }\end{array}$ & $\begin{array}{l}\text { Specific to CT brain images } \\
\text { only }\end{array}$ & $\begin{array}{l}\text { Compresses the CT brain images without the loss of } \\
\text { information }\end{array}$ \\
\hline [45] & $\begin{array}{l}\text { original image is reconstructed } \\
\text { from the compressed image }\end{array}$ & complex system & \\
\hline \multicolumn{4}{|c|}{$\begin{array}{l}\text { C. Positron Emission Tomography Images Compression } \\
\text { Positron Emission Tomography (PET) was the early } \\
\text { and initial investigation procedure to deliver functional }\end{array}$} \\
\hline \multicolumn{4}{|c|}{$\begin{array}{c}\text { TABLE IV } \\
\text { COMPARISON BETWEEN PET TECHNIQUES }\end{array}$} \\
\hline Reference & Advantages & Limitations & Results \\
\hline$[47]$ & High speed processing systems & Sensitive to quality of image & $\begin{array}{l}\text { Compression rate of } 1.7 \% \text { is acquired with } \\
\text { preservation of the image important statistics }\end{array}$ \\
\hline [48] & Real-time implementation & $\begin{array}{l}\text { De-correlation step (ADPCM) } \\
\text { should be improved before } \\
\text { processing }\end{array}$ & $\begin{array}{l}\text { Compression and decompression at } 27 \mathrm{M} \text { Bytes/sec } \\
\text { rate are achieved }\end{array}$ \\
\hline [49] & $\begin{array}{l}\text { Preserves great reliability for the } \\
\text { rebuilt image that can be utilized }\end{array}$ & $\begin{array}{l}\text { Not suitable for medical } \\
\text { tomographic imaging for chunk }\end{array}$ & $\begin{array}{l}\text { The system has robust potential to deliver both } \\
\text { excellence and optimal compression competence }\end{array}$ \\
\hline
\end{tabular}


International Journal of Engineering Trends and Technology (IJETT) - Volume 19 Number 2 - Jan 2015

\begin{tabular}{|c|l|l|l|}
\hline & $\begin{array}{l}\text { in the PACS scheme and for } \\
\text { analysis atmosphere }\end{array}$ & relics sub sequent & when used with medical imaging \\
\hline$[46]$ & $\begin{array}{l}\text { Significantly decreases the } \\
\text { computational difficulty of } \\
\text { clinical image dispensation for } \\
\text { instance production of functional } \\
\text { images }\end{array}$ & $\begin{array}{l}\text { Most of the pixels concerning } \\
\text { the image quality are neglected }\end{array}$ & $\begin{array}{l}\text { Compression of up to 95\% was achieved without } \\
\text { affecting the image quality }\end{array}$ \\
\hline$[50]$ & $\begin{array}{l}\text { Computational efficiency and } \\
\text { noise removal }\end{array}$ & - & - \\
\hline$[51]$ & Easy parallelization & Complex system & - \\
\hline
\end{tabular}

\section{Electroencephalography (EEG) Data Compression}

The electrical nature of the human nervous system has been innovated for more than a century. It is prominent that the variation of the surface potential distribution on the scalp reflects function and activities emerging from the underlying brain. This variation of the surface potential can be recorded by placing an array of electrodes to the scalp, and measuring the voltage between pairs of these electrodes. These measured voltages are then filtered, amplified, and recorded. The resulting data is called the EEG.As per the usefulness, EEG has proved to be an important tool for diagnosis, monitoring and managing various nervous disorders. The electrical activity of brain changes in accordance with various parameters inside and outside environment. A number of severe disorders in human body which were impossible to be traced in early stages are being predicted with help of EEG. However there are certain artifacts which are present in raw EEG recording. Thus, these raw signals are firstly processed with help of mathematical tools in order to make them more and more informative. The informative signal calculated from recordings is known as ERP (event related potential). These ERP data are very specific and changes with every physiological and biological change in human body. Thus the analysis of ERP has got numerous clinical importance's [52]. In other words EEG is the measurement of electrical activities and schedules of the brain through recording electrodes located on the scalp.It has an ability to discover the power, strength and position of electrical deeds in dissimilar brain zones. In the following the most important EEG techniques that have been published are compared in Table 5 .

-Madan et al., 2004, have presented a method that mainly makes use of the process called Power Spectral Density for the EEG data compression [53].

-Sriraam, 2007, has developed another way of lossless compression EEG signal by the use of neural networks. In his method, threshold process is used to detect the errors among the real and predicted signals. A non- uniform manner is used to quantize the threshold error samples. In order to further improve the compression effectiveness and flexibility, arithmetic encoder is used. Single layer neural network, multilayer neural network and Elman network together with two classical adaptive predictors were used in this process. The outcome of this method is measured in sense of compression ratio. Distinction of error limit and quantization step had a great impact on the results and performance of this method [54].
-Aviyente and Selin, 2007, have introduced a method that is based on a Gabor frame and the results show that it is effective for recovery of EEG signals commencing a minor amount of projections [55].

-Sameni et al., 2008, have presented method makes use of Karhunen-Loeve Transforms (KLT) and wavelets. The method proposes redundancy reduction by means of Independent Component Analysis (ICA) in order to discover a further competent code. Consequences obtained from the method by means of ICA, the process of compression, produce extreme lesser reconstruction faults [56].

-Kiong and Marziliano, 2008, have developed method deals with the compression of EEG signals through the process of sampling theory created in favor of signals in the company of finite rate of innovation. The working flow is composed of the creation of non-uniform linear splines which are the results of modeled seizure signals. After that the next step being carried out is the sampling and reconstruction process which is produced on behalf of the signals having limited proportion of improvement. The method shows that the signals of EEG are greatly compressed at the same time protecting their morphologies [57].

-Sriraam and Eswaran, 2008, have introduced a method that study presents a process of the EEG signals compression through lossless process by means of adaptive error modeling structure. The technique makes use of an interpreter and error modeling method. Histogram computation is used in order to predict the error modeling. A heuristic search is applied to find two areas having minimum value. Next context based bias cancellation scheme is utilized to improve the compression process. Results are constructed on the signals of EEG developed under diverse physical and mental circumstances and the performance is appraised in provision of the compression ratio [58].

-K.Srinivasan and M.R. Reddy 2009, have develop a new method for the selection of optimal wavelet for lossless EEG compression for real-time applications. They discussed a wavelet-based lossless compression scheme for EEG signals. For any wavelet based compression scheme, the selection of the type of wavelet and number of decomposition levels is very important task for obtaining effective data compression. Their EEG compression algorithm consists of a preprocessing step involving computation of the multichannel EEG signals, integer lifting wavelet transform and finally setting partition in hierarchical trees (SPIHT) as a wavelet encoder. The optimal wavelet base is chosen based on the compression ratio as well as time taken for encoding the wavelet coefficients by SPIHT 
coder. The algorithm has been tested with normal and abnormal multi-channel EEG data of 8 minutes duration. The issue of selection of optimal wavelet base and decomposition level to achieve higher compression ratio and less encoding delay thus explores the possibility of real-time applications. The maximum compression ratio that can be obtained from this technique is nearly 2.16. Biorthogonal wavelets with vanishing moments around 2 to 4, with a decomposition level of 6 and 7 are considered as an attractive candidate for lossless EEG compression [59].

- Garry et al., 2000, [60] and Higgins et al., 2010 [61], have proposed two EEG compression techniques using JPEG2000.

-In 2011, Mijovi'c et al. proposed new method based on independent component analysis (PCA) as a preprocessing step for data compression of neonatal EEG. In the preprocessing step, data reduction has been carried out using PCA or Singular Value Decomposition (SVD). In this way, they obtain a reduced matrix of uncorrelated sources which keep most of the variance of the data with a lower number of channels. After PCA or SVD, one of the Independent Component Analysis (ICA) algorithms may be optionally used to make the sources independent, and therefore more compressible. Eventually, the sources are compressed, and together with the mixing matrix, they are sent over the link, and reconstructed on the receiver's site using one of the available compressive sensing (CS) reconstruction algorithms. The used ICA algorithm was Second-Order Blind Identification (SOBI). Iterative Hard Thresholding (IHT) was used in the reconstruction stage. Numerical results have showed that this method clearly outperforms the regular compression algorithms, with the speed gain in the reconstruction stage enhanced by $33 \%$ to $50 \%$ [62].

-N. Sriraam, 2012, has developed a high performance lossless compression approach to EEG signals. This has been done using wavelet transform and neural network predictors. The wavelet coefficients representing the EEG signal have been used to train the neural network predictors. The network error residues have been encoded through a combination of entropy and Lempel-Ziv-arithmetic encoders. To improve the compression efficiency, a new context-based error modeling has been studied. The approach provides a compression ratio of 2.99 and compression efficiency of $67 \%$ as well as less encoding time. This could benefit EEG signal telemedicine applications [63].

-K Srinivasan, and J Dauwels, 2013, have proposed multichannel EEG compression method based on wavelet transform and volumetric coding approach to alleviate some of shortcomings of other EEG compression techniques. In particular, they support progressive transmission and exploit the inter- and intra-channel correlation simultaneously, by arranging the multichannel EEG as matrix (image) or tensor (volume). This method guarantees that a maximum amount of distortion is less than the error tolerance in terms of the number of quantization levels. This algorithm consists of two stages: first the multichannel is arranged as matrix or tensor, which is then subjected to lossy compression using waveletbased coder; next the residual of the lossy reconstructed data is quantized and compressed in a lossless fashion. This second step allows to bound the distortion on the residual, and hence also the reconstructed EEG data. Simulation results have shown that the volumetric-based multichannel compression algorithms achieves higher compression than image-based compression algorithms for most EEG data sets, since they utilize the inter-channel correlation effectively [64].

- Pawar and Nandgaonkar, 2013, have proposed EEG compression technique using EZW technique. While compressing EEG data it is very essential to keep all the characteristics of EEG unaffected. So, techniques used for compression of EEG data should be lossless in order to obtain complete EEG signal at the time of reconstruction. Embedded zero tree wavelet is one of the promising method used for compression of data. This method is based on zero-tree encoding. Instead of only wavelet decomposition, this method encodes the wavelet coefficients in zero trees in order to get progressive layer by layer encoding. EZW technique has been adopted as it gives progressive encoding which can be stopped anywhere or depending upon required bit rate. Data compressed using this technique can give good compression ratio [65].

TABLE V

COMPARISON BETWEEN EEG TECHNIQUES

\begin{tabular}{|c|l|l|l|}
\hline Reference & Advantages & Limitations & Results \\
\hline$[60]$ & Signal quality is persevered & - & $\begin{array}{l}\text { Performs efficiently in compressing EEG data } \\
\text { without significant loss in signal fidelity }\end{array}$ \\
\hline$[53]$ & - & $\begin{array}{l}\text { Limited to single occipital and } \\
\text { single frontal station }\end{array}$ & $\begin{array}{l}\text { The normal complete contract associated besides } \\
\text { manual recording of seven sleep accounts is 68.5\% }\end{array}$ \\
\hline$[54]$ & $\begin{array}{l}\text { Fast and reliable in case of } \\
\text { preservation of image details }\end{array}$ & Computationally complex & $\begin{array}{l}\text { The disparity of error boundary and quantization } \\
\text { phase elect the complete compression output }\end{array}$ \\
\hline$[55]$ & Uses small number of data & $\begin{array}{l}\text { Quality of reconstructed signals is } \\
\text { bit affected }\end{array}$ & Results provide acceptable compression outcome \\
\hline$[56]$ & $\begin{array}{l}\text { Slighter quantity of memory is } \\
\text { required }\end{array}$ & $\begin{array}{l}\text { Yields far smaller reconstruction } \\
\text { errors }\end{array}$ & - \\
\hline$[57]$ & $\begin{array}{l}\text { Compressing the EEG signals } \\
\text { with preserving their } \\
\text { morphologies }\end{array}$ & $\begin{array}{l}\text { Reconstructed only 2K Fourier } \\
\text { coefficient with low } \\
\text { reconstruction error }\end{array}$ & $\begin{array}{l}\text { The method outperformed with classical } \\
\text { compression rate with no loss of information and } \\
\text { signal quality }\end{array}$ \\
\hline$[58]$ & Appropriate for the real time & Complexity of the system is a & Compression rate of 3.23 and efficiency of 70\% is \\
\hline
\end{tabular}


International Journal of Engineering Trends and Technology (IJETT) - Volume 19 Number 2 - Jan 2015

\begin{tabular}{|c|c|c|c|}
\hline & broadcasting & drawback & recorded \\
\hline [61] & $\begin{array}{l}\text { Reduces wireless transmission } \\
\text { and saves power }\end{array}$ & $\begin{array}{l}\text { No accepted limit exists for the } \\
\text { maximum PRD }\end{array}$ & $\begin{array}{l}\text { PRDs of up to } 30 \% \text { can be tolerated without overly } \\
\text { affecting the system's performance }\end{array}$ \\
\hline [66] & Less encoding delay & - & $\begin{array}{l}\text { Results showed the reprocessed improved rate- } \\
\text { distortion performance }\end{array}$ \\
\hline [67] & Minimizes power requirements & Computationally complex & $\begin{array}{l}\text { performs well in relation to other published EEG } \\
\text { compression methods }\end{array}$ \\
\hline [59] & $\begin{array}{l}\text { achieve high compression ratio } \\
\& \text { less encoding delay }\end{array}$ & & $\begin{array}{l}\text { The maximum compression ratio that can be } \\
\text { obtained from this technique is nearly } 2.16\end{array}$ \\
\hline [62] & $\begin{array}{l}\text { sources more structured\& more } \\
\text { easily compressible }\end{array}$ & complex & $\begin{array}{l}\text { Outperforms regular compression methods, with the } \\
\text { speed gain enhanced by } 33 \% \text { to } 50 \%\end{array}$ \\
\hline [63] & $\begin{array}{l}\text { efficient high performance } \\
\text { compression method }\end{array}$ & complex & $\begin{array}{l}\text { Compression ratio of } 2.99 \text { with compression } \\
\text { efficiency of } 67 \%\end{array}$ \\
\hline [64] & $\begin{array}{l}\text { bound the distortion on the } \\
\text { residuals }\end{array}$ & Complex & $\begin{array}{l}\text { Achieve high compression ratio compared to other } \\
\text { image-based methods for most data sets }\end{array}$ \\
\hline [65] & $\begin{array}{l}\text { encoding can be stopped upon } \\
\text { required bit rate }\end{array}$ & & Has good compression ratio \\
\hline
\end{tabular}

\section{FUTURE WORK}

The objective of developing new or modifying previously developed image compression techniques is to improve the quantitative performance such as the compression and the signal to noise ratios, to reduce computational time and to explore new data and applications. In [24], colour images have been changed to grey level images to find the representing symbols. After the probabilities of symbols are calculated and arranged in decreasing order.

Then, lower probabilities are merged and Huffman encoding is performed. In this respect, changing the way of arrangement the probabilities or changing Huffman encoder with other one may enhance the performance and reduce the computation capacity. In [25], image compression has been performed using bi-orthogonal filters, so, different bioorthogonal filters and different filtering implementation can be tried.

In [37], Soundarya and Bhavani, have used hybrid coding techniques for the compression of MRI brain images by dividing the image into ROI and Non-ROI. Then, they adopted lossless coding for ROI and lossy coding for NonROI regions, change the way of dividing the image into regions may perform data reduction prior to compression. In [38], Alagendran et al., have split the input image into groups of slices then a 3-D wavelet transform have been applied. After that a 3-D encoding have been carried out. The low frequency coefficients undergo the Huffman coding and the high frequency coefficients undergo the process of quantification [39]. For better performances, mixing different type of wavelet transformation and mixing type of coding techniques can be explored.

Considering the compression of CT images, two issues can be further investigated. In [44], Sepehrband et al. transformed the image, entropy Huffman encoding has been done, bit stream compression has been applied, and inverse processes have been adopted to reconstruct the image. The effect of using other encoding or mixing different encoding method (lossy and lossless) methods can be investigated. In [45], Kumaret al., have used EZW algorithm with improved version of contourlet transform for the significant region and DWT for the insignificant region together with fuzzy C-means based segmentation technique. When the image is of the right type, curvelets provide a representation that is considerably sparser than other wavelet transforms. Therefore compression of different images produced by different modalities can be tested using curvelets and wavelets transform to conclude the transformation that fits each image.

\section{CONCLUSION}

In this paper different approaches and methods which have been developed in the prospect of medical and brain image compression have been reviewed. Compressing an image is basically a process of reducing the size in bytes of images deprived of demeaning the superiority and quality of the image to an objectionable level. The lossy and lossless categories of compression techniques have been discussed. In some application lossy techniques can be used but in other some applications lossless techniques have to be used. Lossy compression is most commonly used to compress multimedia data like audio, video, and still images, especially in applications such as streaming media. The sub-classifications of two different categories of compression have been discussed with emphasize on advantages and disadvantages. After that we have provided a brief overview of some medical image compression techniques and provided descriptive comparison between them. Comparing the performance of compression technique is difficult unless identical data sets and performance measures are used. We have concluded with some direction for the future work so that the quantitative performance for image compression and the computational complexity can be enhanced.

\section{REFERENCES}

[1] Hammudy, H. H., "Image Compression Using Multiwavelet Transform", Iraqi Commission For Computer And Information Institute For Postgraduate Studies, M.Sc. Thesis, Jan 2006.

[2] SalehaMasood, Muhammad Sharif, MussaratYasmin, MudassarRaza and SajjadMohsin. Brain Image Compression: A Brief Survey. Engineering and Technology 5(1): 49-59, 2013.

[3] M.Pratap, N. Kumar, "An Optimized Lossless Image Compression Technique In Image Processing” Institute of Technology Roorkee, Dec. 2012 . 


\section{International Journal of Engineering Trends and Technology (IJETT) - Volume 19 Number 2 - Jan 2015}

[4] Huda Mahmood, "Lossless Image Compression Using Prediction Coding and LZW Scheme", Submitted to College of Science of Baghdad University. 2011

[5] S. Kumar, T. U. Paul, A. Raychoudhury, "Image Compression using Approximate Matching and Run Length", International Journal of Advanced Computer Science and Applications, Vol. 2, No. 6, 2011.

[6] Al-Khoja, S.B., "Wavelet Compression Using Tree and Adaptive Arithmetic Codes", University of Baghdad, M.Sc. Thesis, 2004.

[7] A. B. Watson, "Image Compression Using the Discrete Cosine Transform', Mathematica Journal, 4(1), pp. 81-88, 1994.

[8] Brian A. Wandell, "Foundations of Vision", "https://foundationsofvision.stanford.edu/ chapter-8-multiresolutionimage-representations/", 2014, [accessed 31/12/2014].

[9] A. Jalobeanu, L. Blanc-Feraud, and J. Zerubia.Satellite Image Deconvolution Using Complex Wavelet Packets. Technical report, Institute National de Recherché en Informatiqueet en Automatique, France, 2000 .

[10] Y. Linde, A. Buzo, and R. M. Gray, "An Algorithm for Vector Quantizer Design," IEEE Trans. Communications, vol. 28, no. 1, pp. 84-95, January 1980.

[11] G. Poggi and E. Sasso, "Codebook Ordering Techniques for Address Predictive VQ," in Proc. IEEE Int. Conf. Acoustics, Speech, and Signal Processing '93, Minneapolis, April 27-30 1993, pp. 586-589.

[12] H. Liu and D. Y. Y. Yun, "Self-organizing Finite State Vector Quantization for Image Coding," in Proc. of the Int. Workshop on Applications of Neural Networks to Telecommunications, J. Alspector, R. Goodman, and T. X. Brown, Eds. Hillsdale, NJ: Lawrence Erlbaum Associates, 1993, pp. 176-182.

[13] T. Kohonen, "The Self-organizing Map," Proc. IEEE, vol. 78, no. 9, pp. 1464-1480, September 1990.

[14] J. Li and C. N. Manikopoulos, "Multi-stage Vector Quantization Based on the Self-organization Feature Maps," in SPIE Vol. 1199 Visual Communications and Image Processing IV (1989), 1989, pp. 10461055

[15] S. P. Luttrell, "Hierarchical Vector Quantization," IEE Proceedings (London), vol. 136 (Part I), pp. 405-413, 1989.

[16] T.-C. Lee and A. M. Peterson, "Adaptive Vector Quantization Using a Self-development Neural Network," IEEE J. on Selected Areas in Communications, vol. 8, no. 8, pp. 1458-1471, Oct. 1990.

[17] Ajit Singh, Meenakshi Gahlawat, " Image Compression and its Various Techniques", International Journal of Advanced Research in Computer Science and Software Engineering, Volume 3, Issue 6, June 2013.

[18] Wang, J. and K. Huang, 1996. Medical Image Compression by Using Three-dimensional Wavelet Transformation. Med. Imaging, IEEE Trans., 15(4): 547-554

[19] Rodet, T., P. Grangeat and L. Desbat, 2000. A New Computation Compression Scheme Based on a Multi Frequential approach. Nuclear Science Symposium Conference Record, 2000 IEEE, vol.2, pp. 15/267$15 / 271$.

[20] Hashimoto, M., K. Matsuo, A. Koike, H. Hayashi and T. Shimono, 2004. "CT Image Compression with Level of Interest Image Processing”, 2004.ICIP '04. 2004 International Conference, 5, pp. 3185- 3188

[21] Kanoun, O., M.S. Bouhlel and S. Mezghani, 2006. "Medical Images Adaptive Compression With Statistical Model for Transmission and Archiving", Application to MRI modality. Information and Communication Technologies, 2006. ICTTA '06.2nd, vol.1, pp. 14571462

[22] Shaou-Gang, M., K. Fu-Sheng and C. Shu-Ching, "A Loss less Compression Method for Medical Image Sequences Using JPEG-L Sand Interframe Coding", IEEE 2009

[23] Tzong-Jer, C. and C. Keh-Shih, 2010. A Pseudo Lossless Image Compression Method. Image and Signal Processing (CISP), 2010 3rd International Congress, vol.2, pp. 610-615

[24] Vaishali G. Dubey, Jaspal Singh, “3D Medical Image Compression Using Huffman Encoding Technique”, ECE Department, RIEIT Railmajra, SBSNagar, Punjab, International Journal of Scientific and Research Publications, Volume 2, Issue 9, September 2012

[25] Anju B, Manimurugan S, "An Approach to Medical Image Compression Using Filters Based on Lifting Scheme", IOSR Journal of VLSI and Signal Processing, vol. 1, no. 2 pp. 9-16, Sep-Oct. 2012.
[26] Sandeep Kumar, NitinGoel, Vedpal Singh, AmitChaudhary, NirmalSirohi, Gurbaj Singh, "Fast and Efficient Medical Image Compression Using Contourlet Transform: (FEMI-CCT)", Ganpati Institute of Technology \& Management, India, Open Journal of Computer Sciences, Vol.1, No.1, 7-13, May, 2013

[27] Yilmaz, R. and I. Kilic, 1998. Hierarchical Finite State Vector Quantization for MRI and CT Image Compression. Electrotechnical Conf., 1998. MELECON 98, 9th Mediterranean, vol.1, pp. 77-81.

[28] Midtvik, M. and I. Hovig, 1999. Reversible Compression of MR Images. Medical Imaging, IEEE Trans., 18(9): 795-800.

[29] Cavaro-Menard, C., A. Le Duff, P. Balzer, B. Denizot, O. Morel, P. Jallet and J.J. Le Jeune, Quality Assessment of Compressed Cardiac MRI. Effect of lossy compression on computerized physiological parameters," Inter. Conf., on Image Analysis and Processing, pp. 1034 1037, 1999

[30] Dhouib, D., A. Nait-Ali, C. Olivier and M.S. Naceur, 1994 Comparison of Wavelet Based Coders Applied to 3D Brain Tumor MRI Images. Systems, Signals and Devices, 2009.SSD'09. 6th International Multi- Conference, pp. 1-6, 23-26 March 2009.

[31] Badawy, W., M. Weeks, Z. Guoqing, M. Talley and M.A. Bayoumi, 2002. "MRI Data Compression Using a 3-D Discrete Wavelet Transform". Eng. Med. Bio. Magazine, IEEE, 21(4): 95-103.

[32] Karras, D.A., 2009. "Compression of MRI Images Using the Discrete Wavelet Transform and Improved Parameter Free Bayesian Restoration Techniques". Imaging Systems and Techniques, IEEE Inter. Workshop, pp. 173-178, 2009

[33] Hu, L.L., F. Zhang, Z. Wang, X.F. You, L. Nie, H.X. Wang, T. Song and W.H. Yang, Comparison of the 1HR Relaxation Enhancement Produced by Bacterial Magnetosomes and Synthetic Iron Oxide Nanoparticles for Potential Use as MR Molecular Probes. Appl. Supercond., IEEE Trans., 20(3), pp. 822-825, 2010.

[34] Gornale, S.S., V.T. Humbe, S.S. Jambhorkar, P. Yannawar, R.R. Manza and K.V. Kale, 2007. Multi-Resolution System for MRI (Magnetic Resonance Imaging) Image Compression: A Heterogeneous Wavelet Filters Bank Approach.Computer Graphics, Imaging and Visualization, 2007. CGIV '07, pp. 495-500.

[35] Li, G., J. Zhang, Q. Wang, C. Hu, N. Deng and J. Li, 2006. Application of Region Selective Embedded Zerotree Wavelet Coder in CT Image Compression. Engineering in Medicine and Biology Society, 2005.IEEE-EMBS 2005. 27th Annual International Conference, pp. 6591-6594.

[36] Corvetto, A., A. Ruedin and D. Acevedo, 2010. Robust Detection and Lossless Compression of the Foreground in Magnetic Resonance Images. Data Compression Conference (DCC), pp. 529.

[37] G. Soundarya and S. Bhavani, "Comparison of Hybrid Codes for MR Brain Image Compression",Maxwell Scientific Organization, December 15,2012

[38] Alagendran .B, S. Manimurugan , M. John Justin, "Compression of 3D Medical Image Using EDGE Preservation Technique", International Journal of Electronics and Computer Science Engineering, pp. 802-809, 2012

[39] Seddeq E. Ghrare, and Salahaddin M. Shreef, "Proposed Quality Evaluation of Compressed MRI Medical Images for Telemedicine Applications", World Academy of Science, Engineering and Technology 2012, pp. 568-570

[40] Rhodes, M.L., J.F. Quinn and J. Silvester, 1985. Locally Optimal RunLength Compression Applied to CT Images. Med. Imaging, IEEE Trans., 4(2): 84-90.

[41] Lee, H., Y. Kim, A.H. Rowberg and E.A. Riskin, 1991. 3-D Image Compression for X-ray CT Images Using Displacement Estimation. Data Compression Conference, 1991.DCC '91. pp. 453.

[42] Ju, L.O. and A.K. Seghouane, 2009. False Positive Reduction in CT Colonography Using Spectral Compression and Curvature Tensor Smoothing of Surface Geometry. Biomedical Imaging: From Nano to Macro, 2009. ISBI '09. IEEE International Symposium, pp. 89-92.

[43] Signoroni, A., S. Masneri, A. Riccardi and I. Castiglioni, 2009. Enabling Solutions for an Efficient Compression of PET-CT Datasets Nuclear Science Symposium Conference Record (NSS/MIC), 2009 IEEE, pp. 2747-2751

[44] Sepehrband, F., M. Mortazavi and S. Ghorshi, 2010. Efficient DPCM Predictor for Hardware Implementation of Lossless Medical Brain CT 


\section{International Journal of Engineering Trends and Technology (IJETT) - Volume 19 Number 2 - Jan 2015}

Image Compression. Signals and Electronic Systems (ICSES), 2010 International Conference, pp. 123-126.

[45] S. Kumar1, N. Goel2, V. Singh3, A. Chaudhary4, N. Sirohi5, G. Singh, "Fast and Efficient Medical Image Compression Using Contourlet Transform", Open Journal of Computer Sciences, Vol.1, No.1, pp. 713, May, 2013.

[46] Weidong, C., F. Dagan and R. Fulton, 1998. "Clinical Investigation of a Knowledge-Based Data Compression Algorithm for Dynamic Neurologic FDG-PET Images". Engineering in Medicine and Biology Society, 1998. Proceedings of the 20th Annual International Conference of the IEEE, vol.3, pp. 1270-1273.

[47] Dahlbom, M., K.A. Gardner, A. Chatziioannou and C.K. Hoh, 1994. Whole Body PET Image Compression. Nuclear Science Symposium and Medical Imaging Conference, 1994. IEEE Conference Record, vol. 3, pp. 1394-1398.

[48] Macq, B., M. Sibomana, A. Coppens, A. Bol, C. Michel, K. Baker and B. Jones, 1994. "Lossless Compression for 3D PET". Nucl. Sci., IEEE Trans., 41(4): 1556-1559.

[49] Min-Jen, T., J.D. Villasenor, A. Chatziioannou and M. Dahlbom, 1995. Positron Emission Tomography Compression by Using Wavelet Transform. Nuclear Science Symposium and Medical Imaging Conference Record, 1995, 1995 IEEE, vol.3, pp. 1434-1437.

[50] Zhe, C., B. Parker and D.D. Feng, 2003. "Temporal Compression for Dynamic Positron Emission Tomography via Principal Component Analysis in the Sinogram Domain". Nuclear Science Symposium Conference Record, 2003 IEEE, vol. 4, pp. 2858-2862.

[51] Panins, V.Y., 2008. "Iterative Algorithms for Variance Reduction on Compressed Sinogram Random Coincidences in PET". Nuclear Science Symposium Conference Record, 2008.NSS '08. IEEE, pp. 3719-3725.

[52] Ashish Raj, AkankshaDeo, Mangesh S. Tomar, Manoj Kumar Bandil, "Analysing the Inclusion of Soft Computing Techniques in Denoising EEG Signal”, International Journal of Soft Computing and Engineering, Volume-2, Issue-4, September 2012

[53] Madan, T., R. Agarwal and M.N.S. Swamy, 2004. Compression of Long-term EEG Using Power Spectral Density. Engineering in Medicine and Biology Society, 2004.IEMBS '04. 26th Annual International Conference of the IEEE, pp. 180-183.

[54] Sriraam, N., 2007. "Neural Network Based Near- Lossless Compression of EEG Signals with Non Uniform Quantization". Engineering in Medicine and Biology Society, 2007.EMBS 2007. 29th Annual International Conference of the IEEE, pp. 3236-3240.

[55] Aviyente, S., 2007. "Compressed Sensing Framework for EEG Compression”. Statistical Signal Processing, SSP '07. IEEE/SP 14th Workshop, pp. 181-184, 2007.

[56] Sameni, R., C. Jutten and M.B. Shamsollahi, "Multichannel Electrocardiogram Decomposition Using Periodic Component Analysis,” Biomed. Eng., IEEE Trans., 55(8), pp. 1935-1940, 2008.

[57] Kok-Kiong, P. and P. Marziliano, 2008. "Compression of Neonatal EEG Seizure Signals with Finite Rate of Innovation". Acoustics, Speech and Signal Processing. ICASSP 2008. IEEE International Conference, pp. 433-436, 2008.

[58] Sriraam, N. and C. Eswaran, "An Adaptive Error Modeling Scheme for the Lossless Compression of EEG Signals". Inf. Technol. Biomed., IEEE Trans., 12(5): 587-594, 2008.

[59] K. Srinivasan and M.R. Reddy, "Selection of Optimal Wavelet for Lossless EEG Compression for Real-time Applications", Indian J. of Biomechanics, 2009, pp. 241-245.

[60] H. Garry, M.G. Brian and G. Martin, 2000. Efficient EEG Compression Using JPEG 2000 with Coefficient Thresholding. 2010, UCC, Cork

[61] Higgins, G., S. Faul, R.P. McEvoy, B. McGinley, M. Glavin, W.P. Marnane and E. Jones, 2010a. "EEG Compression Using JPEG2000: How much loss is too much? Engineering in Medicine and Biology Society (EMBC)", Annual IEEE Inter. Conf., pp. 614-617, 2010.

[62] B. Mijovi'c, V. Mati'c, M. De Vos, and S. V. Huffel, "Independent Component Analysis as a Preprocessing Step for Data Compression of Neonatal EEG”, Engineering in Medicine and Biology Society, EMBC, 2011 Annual International Conference of the IEEE, pp. 7316-7319, 2011.

[63] N. Sriraam, “A High-Performance Lossless Compression Scheme for EEG Signals Using Wavelet Transform and Neural Network
Predictors", International J. of Telemedicine and Applications, Vol. 2012, 2012.

[64] K. Srinivasan, J. Dauwels, and M. R. Reddy," Multichannel EEG Compression: Wavelet-Based Image and Volumetric Coding Approach", IEEE J. of Biomedical and Health Informatics, Vo. 17, No. 1, pp. 113-120, Jan. 2013.

[65] Darshana, D. Pawarand, A. B. Nandgaonkar, "EEG Compression Using EZW Technique", International Journal of Science, Engineering and Technology Research (IJSETR), vol. 2, Issue 7, pp. 1509-1512, July 2013.

[66] Srinivasan, K. and R.M. Ramasubba, "Efficient Preprocessing Technique for Real-time Lossless EEG Compression," Elect. Lett, 46(1): 26-27, 2010

[67] Higgins, G., B. McGinley, M .Glavin and E. Jones, "Low Power Compression of EEG Signals Using JPEG2000," 4th International Conf. on Pervasive Computing Technologies for Healthcare, pp. 1-4, 2010.

[68] A. Katharotiya, S. Patel, and M. Goyani, "Comparative Analysis between DCT and DWT Techniques of Image Compression", Journal of Information Engineering and Applications, Vol. 1, No.2, 2011. 